\title{
Subtyping Gambling Acitivities: Case of Korca City, Albania
}

\author{
Prof. Assoc. Dr. Besa SHAHINI \\ Department of Applied Statistics and Informatics,Faculty of Economy, University of Tirana- Albania \\ Tel: +355684018309, besa. shahini@unitir. edu. al besashahini@yahoo.com
}

PhD candidate: Emil FRASHERI

Department of Management, Faculty of Economy, University "Fan S. Noli"

efrasheri@hotmail.co.uk

Abstract

Gambling research has grown dramatically over the past 2-3 decades, however a lack of consensus regarding the risk factors and gambling etiology related to youth problem gambling still remain. So a better understanding of the nature of youth problem gambling could help us to clarify the etiology of gambling problems. Understanding gambling subtypes is necessary to improve our understanding of the etiology of problem gambling. The prediction of problem gambling is related with the participation in gambling activities. It is necessary to obtain a structure of gambling activities, in order to better understand gambling related problems and to treat problems in a more specified manner. The aim of the study was to determine the appropriate structure of gambling activities using factor analysis in a confirmatory framework. Students are a particularly interesting population in which to study gambling. The research utilized a cross-sectional design and self-report questionnaires. The study concludes that the two-factor solution better represents the chance-and skill-based gambling activities. The first factor is most strongly associated with chance-based activities (lottery, bingo, scratch cards). The second factor is most strongly associated with activities that require some degree of skill (poker, roulette, sport bet, racing, etc. ).

Keywords: Gambling, Latent classes, Probability

\section{Introduction}

Problem gambling is being viewed increasingly as a behavioral addiction and has been re-classified as an addictive disorder in the Diagnostic and Statistical Manual of Mental Disorders, Fifth Edition (DSM-5)[1]. Although most people engage in gambling as a recreational activity, some people develop problems that can be linked to this behavior. Situational and structural characteristics of gambling activities may be important moderators of behavior and cognition in gambling and should be taken into account in explanations of gambling behavior. Research has tended to examine gambling as a single entity, incorporating a range of activities that are very different, or looked specifically at individual activities, but, recently, experts have raised concerns about the common practice of lumping together involvement in different activities in studies of the correlates of gambling and have suggested that this practice may be contributing to the contradictory findings that are frequently found in the literature [2]. Sub typing models are thought to have implications for understanding disordered gambling. Identifying different groups could have important implications for prevention and treatment responses. 


\section{Gambling Activities}

Gambling is an umbrella term that covers a number of activities. These activities include electronic gaming (slot) machines (EGMs), betting on horse/dog races, playing scratch cards, playing the lottery, playing keno, playing table games at a casino such as blackjack or roulette, playing bingo for money, betting on sport events, playing cards or other such games for money outside of a casino, and betting on games of skill such as billiards etc. It is clear that these activities are vastly different in terms of their configuration. Gambling activities can be categorized along several dimensions. One commonly drawn distinction is between games that are mainly or entirely based on chance and games that are skill (or perceived skill)-based to a certain extent. Lotteries, scratch cards and slot machines are examples of the first category, whereas poker is an example of the latter. However, while exists a general structure of skill and chance, the distinction is undoubtedly blurred at the level of the game as many gamblers are known to play chance based games with the illusion of control [3]. Slot machine playing is generally acknowledged as involving no skill and leading to an inevitable loss of money.

However, heavy slot machine players privately accept another belief: that their special knowledge of machines will provide a winning edge. Some research [4] suggested that irrational thoughts are commonly present among gamblers and that they may play a central role in maintaining gambling in games of chance. From the other side, sports betting is often characterized as a skill-based form of gambling, but several studies question this by demonstrating that 'experts' in sports betting fail to outperform a chance level of cash return. Similar to adults, adolescent problem gamblers are not a homogenous group. Hence, it is possible that different types of adolescents will engage in different gambling activities. A review of the literature showed that gambling activities are routinely treated as a homogeneous group. Recent research [57] has found that there are two dimensions underlying gambling among adults.

These dimensions distinguish between skill-and chance-based activities. However, another research study [8] among adolescents found that three dimensions underlie the 12 activities surveyed, representing the distinction between three types of gambling activities, specifically those that are chance-based (e. g. , playing poker machines), those that involve gambling on activities which involve some degree of skill to select a winner (e. g. , sports betting), and those which involve gambling on one's own skills (e. g. , gambling on pool). Some individuals are drawn to gambling products such as poker and blackjack that involve an element of skill because they believe they are 'challenging' themselves in addition to competing against other players or the house.

In this sense, individuals might erroneously believe that, they can "improve" their gambling skill through learning and experience, because some games allow to some extent gamblers to attempt to use knowledge of the game to influence or predict the outcome (e. g. , poker, blackjack) which, in turn, could involve a period of regular (financial) losses. Other partially skill-based gambling types are sports betting, which would include horse racing, and speculation on the stock market. Individuals drawn to such gambling types tend to find pleasure in researching the event and testing their own "expertise" in the field while making their bets. Furthermore, these individuals are seeking to enhance the entertainment value of the event or the sport itself by betting on its outcome. In other words, they not only hope their team (or horse) will win but also they will enjoy a windfall from that event.

\section{Measurement Issues Related the Structure of Gambling Activities and Adolescents Disordered Gambling}

Having a set of observed variables (gambling activities' types), some statistical techniques can be applied in discovering which variables in the set form coherent subsets (subtypes) that are relatively independent of one another. Factor analysis attempts to achieve parsimony by explaining the maximum of amount of common variance in a correlation matrix using the smallest number of explanatory constructs (factors or latent variables). In factor analysis only the variance that each observed variable shares with other observed variables is available for analysis [9]. The process of deciding how many factors to keep is called extraction.

We retain only factors with large eigenvalues $(\lambda>1)$. Selection of the number of factors is probably more critical than solution of extraction and rotational techniques. An additional technique is Horn's parallel analysis. Parallel analysis involves 
comparing the size of the eigenvalues with those obtained from a randomly generated data set of the same size. Only those eigenvalues that exceed the corresponding values from the random data set are retained. This approach to identifying the correct number of factors to retain has been shown to be the most accurate, with both Kaiser's criterion and Catell's scree test tending to overestimate the number of factors. After extraction, rotation is used to improve the interpretability and scientific utility of the solution. The use of factor analysis is purely explanatory, usually performed in the early stages of research. It should be used only to guide future hypothesis, or to inform researchers about patterns within data sets. Confirmatory factor analysis (CFA) is associated with theory testing. CFA is much more sophisticated technique used in the advanced stages of the research process where variables are carefully and specifically chosen to reveal underlying processes. Confirmatory factor analysis (CFA) is used to study the relationships between a set of observed variables and a set of continuous latent variables. When the observed variables are categorical, CFA is also referred to as item response theory (IRT) analysis.

After the model has been specified and then estimated, the major question is, "Is it a good model?" One component of a "good" model is the fit between the sample covariance matrix and the estimated population covariance matrix. A good fit is sometimes indicated by a nonsignificant $X^{2}$. A $X^{2}$ statistic is computed based upon the function minimum when the solution has converged. This value is multiplied by $\mathrm{N}-1$ ( $\mathrm{N}=$ number of participants) to yield the $X^{2}$ value, consequently, with large samples, trivial differences between sample and estimated population covariance matrices are often significant. Because of this problem, numerous measures of model fit, which provide often a better gauge of fit, have been proposed. One very rough "rule of thumb" directly related to the $x^{2}$ value is that a good-fitting model may be indicated when the ratio of the $x^{2}$ to the degrees of freedom is less than two.

Fit indices are often used to supplement the $X^{2}$ test to evaluate the acceptability of latent variable models. Since the introduction of model fit indices to the analysis of covariance structures, numerous fit indices based on different rationales have been proposed and studied. Comparative Fit Index (CFI) [10] and Tucker Lewis Index (TLI) [11] are incremental fit indices, measuring the improvement of fit by comparing the hypothesized model with a more restricted baseline model. Values can range from 0-1. For these indices, values above. 90 indicate reasonable fit. Values above. 95 indicate good model fit. CFI has smaller sampling variability than TLI. The Root Mean Square Error of Approximation (RMSEA) is a measure of "discrepancy per degree of freedom" in a model, estimating the lack of fit in a model compared to a perfect (saturated) model. RMSEA has a known distribution and, thus, permits the calculation of confidence intervals. It was suggested [12] that RMSEA values larger than 0.1 are indicative of poor-fitting models, values in the range of 0.05 to 0 . 08 are indicative of fair fit and values less than 0.05 are indicative of close fit. A cut-off value of RMSEA close to 0.06 has been recommended. SRMR and WRMR are residual-based fit indices that measure the (weighted) average differences between the sample and estimated population variances and co-variances. SRMR-the standardized root mean-square residual [13] is an absolute measure of fit. It is defined as the standardized difference between the observed correlation and the predicted correlation.

A cut-off value close to 0.08 has been recommended for SRMR. WRMR- Weighted Root-mean-square Residual [14] is suitable for models where sample statistics have widely disparate variances and when sample statistics are on different scales such as in models with mean and/or threshold structures. It is also suitable with non-normal outcomes. WRMR $\leq 1$. 0 can be used to identify good simple models when $N \geq 500$. Under severe no normality (with continuous variables), SRMR at a cutoff value close to 0.7 , the ML-based TLI and CFI at a cutoff value close to 0.95 and WRMR at a cutoff value close to 1.0 were still applicable when sample size was equal or larger than 500 . Although stringent cut-offs (i. e. , >. 95 for CFI and TLI, <. 06 for RMSEA) have been recommended, and a cut-off lower than 1.0 for WRMR has been identified, a recent research [15] has suggested that, strict adherence to these cut-off values often lead to erroneous results, as factor loadings in social sciences are typically lower. 


\section{Case Study}

The study included 726 students ( $60.5 \%$ females, $39.5 \%$ males) from the University of Korça, ranged from 18 to 23 years old. The survey was conducted between June and July, 2015. Data were collected using a self-reported questionnaire. We focused only on respondents who had played in at least one of the survey gambling activities on at least one occasion during the last 12 months. Participants were given a questionnaire during regular class time assessing their past gambling history, frequency of gambling behavior, types and number of gambling activities in which they engaged. The total time required for completion of the questionnaire was approximately 15 minutes.

Data was analyzed using SPSS and Mplus software packages. The term "gambling" was used to describe a widely varying array of activities. These activities included lotteries, bingo, scratch cards, sport betting, EGMs, race, poker, roulette, internet gambling, dice and other gambling activities outside of a casino. For each of the eleven gambling activities, respondents answered on a six-alternative scale: 0 - never; 1- less than once monthly; 2- less than once weekly; 3- 1-2 times a week; 4- 3-5 times a week; 5- 6-7 times a week. The goal of the study was to determine the appropriate structure of gambling activities among the students using the appropriate statistical techniques. An exploratory factor analysis (EFA) in a confirmatory framework was conducted on the eleven gambling activities with both, orthogonal and oblique rotations using SPSS version 20 and Mplus software packages.

The sample size of 726 cases was considered as very good [16] for factor analysis. Prior to performing EFA, the suitability of data for factor analysis was assessed using several tests. Most of the values in the negative anti-image correlation matrix were small. The Kaiser-Meyer-Olkin value was. 822, exceeding the recommended value of. 6 [17] and Bartlett's Test of Sphericity reached statistical significance, supporting the factorability of the correlation matrix. The factor analysis revealed the presence of three factors with eigenvalues exceeding one, explaining $31.375 \%, 14.085 \%$ and $9.296 \%$ of the variance respectively. (Table 1)

Table 1: Total Variance Explained in an extraction method with Principal Axis Factoring.

\begin{tabular}{|c|c|c|c|c|c|c|c|}
\hline \multirow[t]{2}{*}{ Factor } & \multicolumn{3}{|c|}{ Initial Eigenvalues } & \multicolumn{3}{|c|}{ Extraction Sums of Squared Loadings } & \multirow{2}{*}{$\begin{array}{l}\text { Rotation Sums of } \\
\text { Squared Loadings } \\
\text { Total }\end{array}$} \\
\hline & Total & $\%$ of Variance & Cumulative $\%$ & Total & $\%$ of Variance & Cumulative $\%$ & \\
\hline $\begin{array}{l}1 \\
2 \\
3 \\
4 \\
5 \\
6 \\
7 \\
8 \\
9 \\
10 \\
11\end{array}$ & $\begin{array}{l}3.451 \\
1.549 \\
1.023 \\
833 \\
797 \\
734 \\
630 \\
534 \\
515 \\
477 \\
457\end{array}$ & \begin{tabular}{|l}
31.375 \\
14.085 \\
9.296 \\
7.572 \\
7.245 \\
6.676 \\
5.729 \\
4.851 \\
4.681 \\
4.340 \\
4.150
\end{tabular} & $\begin{array}{l}31.375 \\
45.460 \\
54.757 \\
62.329 \\
69.574 \\
76.250 \\
81.979 \\
86.830 \\
91.510 \\
95.850 \\
100.000\end{array}$ & $\begin{array}{l}2.904 \\
967 \\
428\end{array}$ & $\begin{array}{l}26.398 \\
8.789 \\
3.890\end{array}$ & $\begin{array}{l}26.398 \\
35.187 \\
39.077\end{array}$ & $\begin{array}{l}\text { 2. } 668 \\
\text { 1. } 355 \\
\text { 1. } 221\end{array}$ \\
\hline
\end{tabular}

Source: Authors' calculations using SPSS.

Facing this situation, a parallel analysis was performed using SPSS syntax developed by O'Connor (2000). Parallel analysis is considered one of the most accurate and underutilized methods to determine the number of retainable factors [18]. Factors were retained whenever the eigenvalues from the original data for a given factor exceed the eigenvalues corresponding to the desired percentile (usually the 95th) of the distribution of random data eigenvalues.

The eigenvalues (computed from 100 random data sets) were used for comparison against eigenvalues computed from the original data set. The column of interest to us was the one in which eigenvalues were listed under the heading "Percentile." These values correspond to the $95^{\text {th }}$ percentile of the eigenvalues for each factor that were obtained from the 100 randomlygenerated data sets. We found that only two factor eigenvalues in this column were less than the factor eigenvalues from the original matrix, consequently, we can vest confidence that only the first two factors should be retained. (Table 2)

However, an inspection of the scree plot did not reveal a clear break after the third factor. (Graph 1) 
Graph 1: Scree plot for factor extraction.

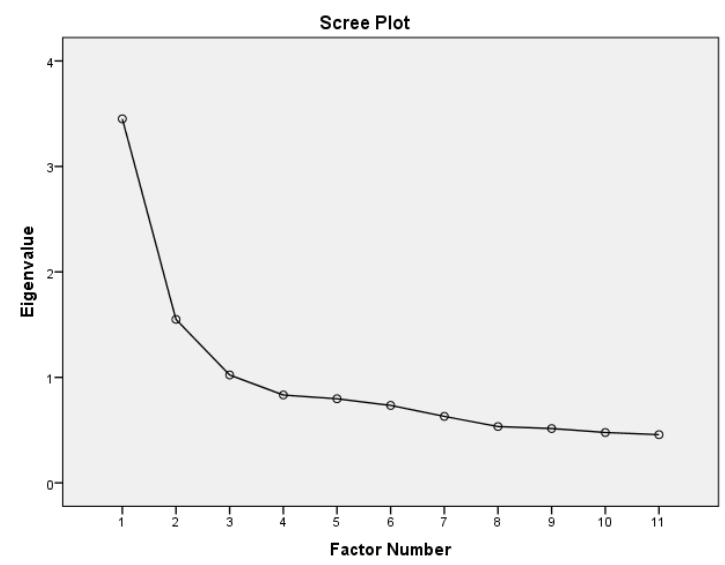

Source: Authors' calculations using SPSS.

Table 2:Comparison of Factor Analysis and Parallel Analysis eigenvalues.

Root Raw Data Means Percentile

1. 000000 3. 4512291.2003331 .252757

2. 000000 1. 5494011.1445021 .182812

3. 000000 1. 0226051.1012251 .132791

4. 000000.832928 1. 0639021.090765

5. 000000.7969741 .0298921 .055062

6. 000000.734369 .9964301 .020813

7. 000000.630160 .962671 .986745

8. 000000.533592 .930471 .955453

9. 000000.514863 .896816 .924672

10. 000000.477368 .860240 .892883

11. 000000.456510 .813517 .850535

Source: Authors' calculations.

The decision between orthogonal and oblique rotation was made as soon as the number of reliable factors was apparent.

Perhaps the best way to decide between orthogonal and oblique rotation was to request oblique rotation with the desired number of factors and look at the correlations among factors [9]. Looking at the correlation matrix with two factors and oblique rotation, the level of correlation matrix (.306) was considered borderline between accepting an orthogonal solution versus dealing with the complexities of interpreting an oblique solution so, the simpler, orthogonal, solution with varimax rotation and two factors was evaluated.

Factors were interpreted through their factor loadings from table 3 . After orthogonal rotation, the values in the loading matrix are correlations between variables and factors. It was decided to use a loading of. 45 . Loadings under. 45 ( $20 \%$ of variance) were replaced by zero. (Table 3 )

Table 3: Rotated Factor Matrix using Principal Axis Factoring with Varimax rotation. 


\begin{tabular}{|l|l|l|}
\hline \multirow{2}{*}{} & \multicolumn{2}{l|}{ Factor } \\
\cline { 2 - 3 } & 1 & 2 \\
\hline Poker & 714 & \\
EGM & 642 & 000 \\
Roulette & 630 & 000 \\
Sport bet & 623 & \\
Internet betting & 586 & \\
Race & 548 & 000 \\
Dice & 458 & 000 \\
Other & .000 & \\
Bingo & & 690 \\
Scratch cards & & 583 \\
Lottery & .000 & 474 \\
\hline
\end{tabular}

Source: Authors' calculations using SPSS.

To interpret a factor, we tried to understand the underlying dimension that unifies the group of variables, loading on it. The two factors reflected the distinction between chance-based and skill or perceived skill-based gambling activities.

The first factor was most strongly associated with skill or perceived skill-based activities with some activities such as poker and roulette, which involve higher degree of skill, to have the highest loadings with the factor and some other partially skill or perceived skill-based activities such as sport bet, racing to have lower correlation with the same factor.

We labeled this factor SKILL.

The second factor was most strongly associated with chance-based activities such as bingo, scratchcards and was labeled CHANCE. The variable related to gambling activities outside of a casino such as cards play or billiards, labeled as OTHER, resulted to have a loading of. 147 on the first factor and was replaced with zero.

Most of the ordered categorical variables used in this analysis (7) resulted to have a no normal distribution with some of them having values of skewness and kurtosis greater than two and seven respectively. So, the multivariate normality assumption underlying the ML estimation was violated. Facing with this situation, some research [19], recommend concern, especially for kurtosis.

Consequently, adjustments to the $x^{2}$ and standard errors based on a weight matrix derived from an estimate of multivariate kurtosis were made. Mplus prints this kurtosis adjustment, referred to as the "scaling correction factor" (SCF). When modeling ordered categorical data with at least five categories, treating the data as continuous in nature and employing ML estimation with the Satora-Benttler scaling methods appears to work well and has been recommended in this situation [20].

Taking into account that our variables were ordered categorical with six categories, the best recommendation was: 1) to treat data as continuous and to use S-B scaling methods with ML estimation or 2) to treat data as categorical and to use DWLS estimation.

One could employ and report findings from both S-B scaling and robust DWLS estimation [21].

Having so, an exploratory factor analysis in Mplus using two robust estimators (WLSMV and MLR) and GEOMIN OBLIQUE rotation, was performed. Running EFA for one to three factor models in Mplus, ordered categorical variables for the WLSMV estimator and continuous variables for the MLR estimator were used. The model with two factors resulted to have the best fit indices with both estimators as in tables below.

Table 4: EFA with one to three factors using WLSMV estimator.

\begin{tabular}{|l|l|l|l|}
\hline$X^{2}$ test of model fit & 508.355 & 107.689 & 83.212 \\
\hline d. f. & 44 & 34 & 25 \\
\hline p-value & .0000 & .0000 & .0000 \\
\hline RMSEA & .121 & .055 & .057 \\
\hline \multicolumn{2}{|c|}{78} & \\
\hline
\end{tabular}




\begin{tabular}{|l|l|l|l|}
\hline $90 \%$ C. I. for RMSEA & .111 .130 & .043 .066 & .043 .070 \\
\hline CFI & .777 & .965 & 972 \\
\hline TLI & .721 & .943 & .938 \\
\hline SRMR & .091 & .052 & .039 \\
\hline
\end{tabular}

Source: Authors' calculations using Mplus.

Table 5: EFA with one to three factors using MLR estimator.

\begin{tabular}{|l|l|l|l|}
\hline Fit indices & One factor & Two factors & Three factors \\
\hline Nr. of parameters & 33 & 43 & 52 \\
\hline$x^{2}$ test of model fit & 187.347 & 65.794 & 95.502 \\
\hline d. f. & 44 & 34 & 25 \\
\hline p-value & .0000 & .0009 & .0000 \\
\hline SCF for MLR & 1.926 & 2.014 & 0.947 \\
\hline RMSEA & .067 & .036 & .062 \\
\hline $90 \%$ C. I. for RMSEA & .057 .067 & .022 .049 & .049 .076 \\
\hline CFI & .806 & .975 & .904 \\
\hline TLI & .757 & .931 & .790 \\
\hline SRMR & .071 & .032 & .026 \\
\hline AIC & 21172.018 & 20963.664 & 20939.573 \\
\hline BIC & 21323.407 & 21160.929 & 21178.125 \\
\hline SSABIC & 21218.622 & 21024.390 & 21013.010 \\
\hline
\end{tabular}

Source: Authors' calculations using Mplus.

Using the WLSMV estimator, (Table 4) the models with two and three factors were comparable, with the two-factor model to have lower RMSEA and greater TLI, whereas the three-factor model had better CFI and SRMR. However, taking into account the parsimony, the two-factor model was more preferable. Using the MLR estimator (Table 5), the two-factor model performed better in most of the fit indices (RMSEA, CFI, TLI, BIC) and the ratio of $\chi^{2}$ test of model fit divided by the degrees of freedom was less than two, reinforcing our previous judgment. In order to test the proposed model, CFA was performed, using different estimators, in Mplus 6. 12. A two-factor model was hypothesized: a SKILL factor (with poker, roulette, EGM, sport bet, race, internet and dice as indicators) and a CHANCE factor (with lottery, bingo and scratchcards serving as indicators). The goodness of fit was evaluated using a p-value of $X^{2}$ of. 05 for the test of close fit. Additional fit indices included CFI, TLI, RMSEA, SRMR and WRMR (Table 6).

The ICs, considered in this study, were the commonly used AIC, BIC, and adjusted BIC. Comparing across all the models and sample sizes, there seems to be strong evidence that the BIC is the best of the ICs considered [22]. An examination of fit indices showed that the two factor solution, using robust estimators, had an acceptable fit, as demonstrated by the RMSEA, CFI, TLI and SRMR. The model $X^{2}$ with all the estimators resulted to be significant, but with MLMV it was also less than two times the model degrees of freedom. This ratio gives a very rough indication that the model may fit the data. The results from CFA, using different robust estimators, revealed that, TLI, and CFI performed reasonably well with categorical model robust estimation (WLSM and WLSMV).

These results are consistent with previous research [23-24]. RMSEA performed better with ML estimators with values less than. 05 to indicate a good fit, whereas using DWLS estimators, the values of RMSEA were indicators of an acceptable fit. The values of SRMR were well below the cut-off value of. 08, indicating a good fit. WRMR values were greater than one with all the estimators. This is contrary to some previous recommendations [25], but we should not forget that, WRMR is an experimental fit statistic and could be ignored.

Table 6: CFA goodness-of-fit information using different robust estimators.

\begin{tabular}{|l|l|l|l|l|l|l|}
\hline Fit information & $\begin{array}{l}\text { Three factors } \\
\text { MLR }\end{array}$ & $\begin{array}{l}\text { Two factors } \\
\text { MLMV }\end{array}$ & $\begin{array}{l}\text { Two factors } \\
\text { MLM }\end{array}$ & $\begin{array}{l}\text { Two factors } \\
\text { MLR }\end{array}$ & $\begin{array}{l}\text { Two factors } \\
\text { WLSM }\end{array}$ & $\begin{array}{l}\text { Two factors } \\
\text { WLSMV }\end{array}$ \\
\hline Obs. D. V. & Continuous & Continuous & Continuous & Continuous & $\begin{array}{l}\text { Ordered } \\
\text { categorical }\end{array}$ & $\begin{array}{l}\text { Ordered } \\
\text { categorical }\end{array}$ \\
\hline
\end{tabular}




\begin{tabular}{|l|l|l|l|l|l|l|}
\hline $\begin{array}{l}\text { Nr. } \\
\text { parameters }\end{array}$ & 35 & 31 & 31 & 31 & 61 & 61 \\
\hline $\begin{array}{l}\text { p-value } \\
\chi^{2} \text { test of model } \\
\text { fit }\end{array}$ & .0000 & .0006 & .0000 & .0000 & .0000 & .0000 \\
\hline d. f. & 42.237 & 67.351 & 75.949 & 78.160 & 181.139 & 157.643 \\
\hline $\begin{array}{l}\text { SCF for MLR, } \\
\text { MLM }\end{array}$ & 1.927 & 34 & 34 & 34 & 34 & 34 \\
\hline AIC & 20992.896 & 19197.287 & 19197.287 & 19197.287 & - & - \\
\hline BIC & 21153.460 & 19339.501 & 19339.501 & 19339.501 & - & - \\
\hline SSABIC & 21042.324 & 19241.067 & 19241.067 & 19241.067 & - & - \\
\hline RMSEA & .041 & .037 & .041 & .042 & .077 & - \\
\hline 90\% C. I. & .029 .052 & .024 .050 & .029 .054 & .030 & .066 & .071 \\
\hline CFI & .932 & .924 & .936 & .938 & .960 & .060 \\
\hline TLI & .911 & .900 & .916 & .918 & .947 & .921 \\
\hline SRMR & .047 & .048 & .048 & .048 & - & - \\
\hline WRMR & - & 1.216 & 1.216 & - & 1.094 & 1.248 \\
\hline
\end{tabular}

Source: Authors' calculations using Mplus.

\section{Conclusions}

The result of the study revealed that, gambling activities are not a homogenous group, finding that, there are two dimensions underlying gambling among adolescents. These dimensions distinguish between skill-and chance-based activities. This finding reinforced previews research among adults. Inconsistent with other research among adolescents, this study found no differentiation between the skill-based gambling activities.

This can happen because this differentiation is slightly unclear and the types of activities can be specific for each country. In this study, based on a previous recommendation, several psychometric techniques were implemented using two types of robust estimators, treating the observed variables not only as ordered categorical, but, also, as continuous. This combination of psychometric techniques may be helpful to draw better conclusions in gambling-related future studies.

Literature

[1] American Psychiatric Association (APA). Diagnostic and Statistical Manual of Mental Disorders (DSM-5). Arlington, VA: American Psychiatric Publishing; 2013.

[2]Griffiths, M. (2013). Trait expectations: Is there a "gambling personality?" Retrieved from http://www. psychologytoday. com/blog/in-excess/201302/trait-expectations-0.

[3] Wohl, M. J. A. , \& Enzle, M. E. (2002). The deployment of personal luck: Sympathetic magic and illusory control in games of pure chance. Personality and Social Psychology Bulletin, 28, 1388-1397.

[4] Gaboury, A. \& Ladouceur, R. (1988). Irrational thinking and gambling. In W. R. Eadington (Ed. ), Gambling Research. " Proceedings of the Seventh International Conference on Gambling and Risk Taking, vol. 3 (pp. 142-163). Reno, Nevada: University of Nevada-Reno

[5]Young, M. , \& Stevens, M. (2009). Player preferences and social harm: An analysis of the relationships between player characteristics, gambling modes, and problem gambling. International Journal ofMental Health and Addiction, 7 , 262-279.

[6] Stevens, M. , \& Young, M. (2010). Who plays what? Participation profiles in chance versus skill-based gambling. Journal of Gambling Studies, 26, 89-103

[7] Holtgraves, T. (2009b). Gambling, gambling activities, and problem gambling. Psychology of Addictive Behaviors, 23, 295-302 
[8] Boldero, J. M. , Bell, R. C. , \& Moore, S. M. (2010). Do gambling activity patterns predict gambling problems? A latent class analysis of gambling forms among Australian youth. International Gambling Studies, 10.

[9] Tabachnick, B. G. \& Fidell, L. S. (2007). Using multivariate statistics, fifth edition, 635, 646.

[10] Bentler, P. M. (1988). Comparative fit indexes in structural models. Psychological Bulletin, 107, 238-246.

[11] [Tucker and Lewis, 1973] L. R. Tucker and C. Lewis. A reliability coefficient for maximum likelihood factor analysis. Psychometrika, pages 1-10, 1973.

[12] Browne MW, Cudeck R. Alternative Ways of Assessing Model Fit. In: Bollen K, Long J, editors. Testing Structural Equation Models. Sage; Newbury Park, CA: 1993. pp. 136-162.

[13] Joreskog, K. , \& Sorbom, D. (1981). LISREL V: Analysis of linear structural relationships by the method of maximum likelihood. Chicago: National Educational Resources.

[14] Muthén LK, Muthén BO. MPlus User's Guide, $2^{\text {nd }}$ Version. Muthén \& Muthén; Los Angeles: 1998-2001.

[15] Perry, J. L. , Nicholls, A. R. , Clough, P. J. , \& Crust, L. (2015). Assessing model fit: Caveats and recommendations for confirmatory factor analysis and exploratory structural equation modeling

[16] Comfrey, AL and Lee, HB. (1992). A First Course in Factor Analysis. Hillsdale, NJ: Lawrence Erlbaum Associates

[17] Kaiser HF. A Second-Generation Little Jiffy. Psychometrika. 1970; 35(4):401-15.

[18] Fabrigar, L. R. , Wegener, D. T. , MacCallum, R. C. , \& Strahan, E. J. (1999). Evaluating the use of exploratory factor analysis in psychological research. Psychological Methods, 4(3), 272-299.

[19] West, S. G. , Finch, J. F. \& Curran, P. J. (1995). Structural equation models with nonnormal variables. Problems and remedies. In R. H. Hoyle (Ed. ). Structural equation modeling: Concepts, issues and applications (pp. 56-75). Newbury Park, CA: Sage

[20] Rhemtulla M, Brosseau-Liard PE, Savalei V (2012). When can categorical variables be treated as continuous? A comparison of robust continuous and categorical SEM estimation methods under suboptimal conditions. Psychol Methods, 17, 354.

[21] Finney, S. J. , \& DiStefano, C. (2013). Nonnormal and categorical data in structural equation modeling. In G. R. Hancock \& R. O. Mueller (Eds. ), Structural equation modeling: A second course (2nd ed. , pp. 439-492). Charlotte, NC: Information Age Publishing, Inc.

[22] Jedidi, K. , Jagpal, H. S. and DeSarbo, W. S. (1997) 'Finite-mixture structural equation models for response-based segmentation and unobserved heterogeneity'. Marketing Science, 16 (1): 39-59.

[23] Beauducel, A. , \& Herzberg, P. Y. (2006). On the performance of maximum likelihood versus means and variance adjusted weighted least squares estimation in CFA. Structural Equation Modeling, 13, 186 -203.

[24] Hutchinson, S, R. , \& Olmos, A (1998). Behavior of descriptive fit indexes in confirmatory factor analysis using ordered categorical data. Structural Equation Modeling: A Multidisciplinary Journal, 5, 344-364.

[25] Yu, C. -Y. \& Muthén, B. (2002). Evaluation of model fit indices for latent variable models with categorical and continuous outcomes. Paper presented at the annual meeting of the American Educational Research Association, New Orleans, LA 